\title{
Predictions of the Geometries and Fluorescence Emission Energies of Oxyluciferins
}

\author{
By Tianxiao Yang and John D. Goddard \\ Department of Chemistry \\ University of Guelph \\ Guelph, Ontario, Canada, N1G 2W1
}

The optimized cartesian coordinates of anionic keto- and enol-forms of $\mathrm{OxyLH}_{2}(-1)$ by CASSCF $(8,8)$.

$\begin{array}{lrrr}\text { S } 1 \text { state of enol-s-cis(-1) } & & & \\ \mathrm{C} & -0.42049671 & 0.08925657 & 0.00009967 \\ \mathrm{C} & -1.85337907 & 0.02598640 & -0.00002061 \\ \mathrm{C} & -4.24435393 & -0.57997495 & -0.00060450 \\ \mathrm{C} & -3.92843601 & 0.74078797 & -0.00011562 \\ \mathrm{C} & 1.65053594 & 0.87480167 & 0.00040878 \\ \mathrm{C} & 1.97501675 & -0.46425494 & 0.00005880 \\ \mathrm{C} & 3.32226194 & -0.92925002 & -0.00001751 \\ \mathrm{C} & 4.38933441 & 0.03102097 & 0.00055276 \\ \mathrm{C} & 2.69973415 & 1.82133801 & 0.00075097 \\ \mathrm{C} & 4.00377037 & 1.42760463 & 0.00089546 \\ \mathrm{~N} & -2.62684560 & 1.09093254 & 0.00021721 \\ \mathrm{~N} & 0.27072569 & 1.18012094 & 0.00039231 \\ \mathrm{~S} & -2.75566911 & -1.49972317 & -0.00043301 \\ \mathrm{~S} & 0.52030832 & -1.41944082 & -0.00029681 \\ \mathrm{H} & 3.56445333 & -1.97505920 & -0.00051940 \\ \mathrm{H} & 2.44222569 & 2.86617824 & 0.00088983 \\ \mathrm{H} & 4.79962215 & 2.15102073 & 0.00126846 \\ \mathrm{H} & -4.35584295 & 2.54538518 & 0.00040165 \\ \mathrm{H} & -5.20996801 & -1.03670268 & -0.00119660 \\ \mathrm{O} & -4.84778850 & 1.73379547 & 0.00002658 \\ \mathrm{O} & 5.58822745 & -0.33766341 & 0.00074419\end{array}$

$\mathrm{S}_{0}$ state of enol-s-cis(-1)

$\mathrm{C}$

$\begin{array}{lll}-0.40749625 & 0.01922515 & -0.00012026\end{array}$ 


$$
\begin{aligned}
& \text { C } \\
& \text { C } \\
& \text { C } \\
& \text { C } \\
& \text { C } \\
& \text { C } \\
& \text { C } \\
& \text { C } \\
& \text { C } \\
& \text { N } \\
& \text { N } \\
& \text { S }
\end{aligned}
$$

C

C

C

C

C

C

C

$\mathrm{N}$

$\mathrm{N}$

$\mathrm{S}$

$\mathrm{H}$

$\mathrm{H}$

$\mathrm{H}$

$\mathrm{H}$

$\mathrm{H}$

$\mathrm{O}$

$\mathrm{O}$

$\mathrm{S}_{1}$ state of enol-s-twist(-1)

$\mathrm{C}$

C

C

C

C

C

C

C

C

C

$\mathrm{N}$

$\mathrm{N}$

S

$\mathrm{S}$

$\mathrm{H}$

$\mathrm{H}$

$\mathrm{H}$

$\mathrm{H}$

$\mathrm{H}$

$\mathrm{O}$

$\mathrm{O}$

-1.86549116
-4.25377500
-3.92820380
1.63627505
2.02227244
3.32077433
4.39750700
2.65710658
3.96904703
-2.59543161
0.29231275
-2.77820875
0.54596304
3.59538793
2.38151823
4.74855129
-4.30470250
-5.22395848
-4.81005745
5.58404566

0.02051326

$-0.55092685$

0.75860576

0.85444039

$-0.51640024$

$-0.93341940$

0.05665028

1.81552598

1.44843639

1.07442966

1.10177918

$-1.48014388$

$-1.49096615$

$-1.97320235$

2.85762885

2.19067995

2.58015838

$-0.99795836$

1.77663233

$-0.27552822$

0.40347765

1.85414320

4.13448770

4.02247721

$-1.65255021$

$-1.95840169$

$-3.30070442$

$-4.41611799$

$-2.75369856$

$-4.04104504$

2.74921650

$-0.32302215$

2.55121784

$-0.49774549$

5.03432062

5.84873504

$-3.52873711$

$-2.52065375$

$-4.84608906$

5.01580661

$-5.57817591$
0.05855180

$-0.08252675$

0.23428110

0.81859951

$-0.52567373$

$-1.01458702$

$-0.09756005$

1.72111761

1.29256128

0.30822484

1.17671020

$-0.30273791$

$-1.45862322$

$-0.19795526$

0.42523844

$-2.05115232$

2.76341347

1.98721728

0.48544694

$-0.49865887$
0.07726964
$-0.00014122$

$-0.00000377$

$-0.00022321$

0.00050725

$-0.00011805$

$-0.00017696$

0.00037177

0.00114445

0.00116431

$-0.00028343$

0.00046148

$-0.00016416$

$-0.00082118$

$-0.00065882$

0.00161142

0.00175473

$-0.00054917$

$-0.00000892$

$-0.00043591$

0.00019222

$-0.37794534$

$-0.17184987$

0.74702579

$-0.54594204$

$-0.04302252$

$-0.22471710$

$-0.18959280$

0.04312111

0.17283858

0.21604497

$-1.06741893$

$-0.04192172$

1.42814530

$-0.42240444$

1.31807587

$-0.97634460$

$-0.35083113$

0.30550845

0.37984670

$-1.42184128$

0.07171501 
$\mathrm{S}_{0}$ state of enol-s-twist(-1)

C
C
C
C
C
C
C
C
C
N
N
S
S
H
H
H
H
H
O
O

$\begin{array}{rrr}0.39546002 & 0.02661456 & -0.01620048 \\ 1.87385045 & 0.03899329 & 0.04045956 \\ 4.21764099 & -0.09785080 & 0.68527368 \\ 3.96717549 & 0.26244861 & -0.57749395 \\ -1.66926758 & 0.80155276 & 0.06024384 \\ -2.00553600 & -0.55457070 & -0.13193310 \\ -3.30307186 & -1.00472345 & -0.21540830 \\ -4.40659153 & -0.07309936 & -0.10263166 \\ -2.71633812 & 1.72295870 & 0.17230241 \\ -4.01912644 & 1.30179886 & 0.09383876 \\ 2.64060930 & 0.33553199 & -0.94937305 \\ -0.31233625 & 1.08594357 & 0.12621103 \\ 2.71868066 & -0.37131822 & 1.52170268 \\ -0.50279256 & -1.48681009 & -0.23714216 \\ 5.16925571 & -0.21595761 & 1.16219028 \\ 5.73111045 & 0.49309961 & -1.20706299 \\ -3.53686130 & -2.04451276 & -0.36149448 \\ -2.48165487 & 2.76439209 & 0.31965916 \\ -4.82248355 & 2.01389993 & 0.18009782 \\ 4.84950353 & 0.56101902 & -1.54519701 \\ -5.59028555 & -0.44025299 & -0.16955206\end{array}$

$\mathrm{S}_{1}$ state of enol-s-trans(-1)

$\begin{array}{lrrr}\mathrm{C} & 0.12281108 & 0.00031776 & 0.02952110 \\ \mathrm{C} & 0.05988786 & 0.00020128 & 1.45317232 \\ \mathrm{C} & 0.53138689 & -0.00029186 & 3.85618876 \\ \mathrm{C} & -0.77379368 & -0.00018052 & 3.47927671 \\ \mathrm{C} & 0.90224878 & 0.00017061 & -2.04193830 \\ \mathrm{C} & -0.44175209 & 0.00025795 & -2.34781653 \\ \mathrm{C} & -0.91887262 & 0.00016798 & -3.69180950 \\ \mathrm{C} & 0.02914947 & 0.00005642 & -4.77050052 \\ \mathrm{C} & 1.83680874 & 0.00003778 & -3.09995803 \\ \mathrm{C} & 1.42943793 & 0.00003861 & -4.40070255 \\ \mathrm{~N} & -1.05355028 & 0.00012888 & 2.15872189 \\ \mathrm{~N} & 1.21645658 & 0.00023902 & -0.66202155 \\ \mathrm{~S} & 1.52889633 & 0.00013524 & 2.41663811 \\ \mathrm{~S} & -1.38872689 & 0.00044332 & -0.88569430 \\ \mathrm{H} & 0.93276591 & -0.00048821 & 4.84650009 \\ \mathrm{H} & -2.60069658 & -0.00021695 & 3.81913974 \\ \mathrm{H} & -1.96729477 & 0.00019135 & -3.92298392 \\ \mathrm{H} & 2.88481091 & -0.00006552 & -2.85482420\end{array}$


$\mathrm{H}$
$\mathrm{O}$
$\mathrm{O}$

$\mathrm{S}_{0}$ state of enol-s-trans(-1)

C
C
C
C
C
C
C
C
C
N
N
S
S
H
H
H
H
H
O
O

$\mathrm{S}_{1}$ state of keto-s-cis(-1)

C

C

C

C

C

C

C

C

C

C

$\mathrm{N}$

$\mathrm{N}$

$\mathrm{S}$

$\mathrm{S}$

$\mathrm{H}$
2.14465932
-1.81310296
-0.35475392

0.00004426

0.00808550

0.00582338

0.57908319

$-0.73226008$

0.86194799

$-0.50996040$

$-0.92009559$

0.07278286

1.82708575

1.46374164

$-1.05084303$

1.09897975

1.50997397

$-1.49389761$

1.02677466

$-2.55450547$

$-1.95895027$

2.86867499

2.20808778

$-1.74830822$

$-0.25544480$

$$
\begin{array}{r}
0.42183957 \\
1.86760504 \\
4.30615120 \\
3.94566965 \\
-1.65825845 \\
-1.95544150 \\
-3.30322591 \\
-4.38633076 \\
-2.72414714 \\
-4.02014067 \\
2.60550811 \\
-0.28086941 \\
2.73607677 \\
-0.49320354 \\
4.89245630
\end{array}
$$

$-0.00032692$

$-0.00001648$

$\begin{array}{rr}0.00008649 & 0.00411505 \\ 0.00000462 & 1.45290871 \\ -0.00017233 & 3.82942776 \\ -0.00014372 & 3.51470577 \\ 0.00008572 & -2.03039410 \\ 0.00013352 & -2.41644408 \\ 0.00019652 & -3.71743366 \\ 0.00018356 & -4.79134324 \\ 0.00006424 & -3.04660169 \\ 0.00007515 & -4.35959159 \\ -0.00004946 & 2.17892698 \\ 0.00011103 & -0.68336867 \\ -0.00004515 & 2.35012698 \\ 0.00006752 & -0.94358213 \\ -0.00014280 & 4.79967404 \\ -0.00010205 & 3.89968679 \\ 0.00028794 & -3.99605404 \\ 0.00002972 & -2.76841797 \\ 0.00004668 & -5.13708880 \\ -0.00013313 & 4.40013293 \\ 0.00025992 & -5.97906401\end{array}$

4.34774616

$-5.96441237$

$-5.97906401$

$\begin{array}{rr}0.13511632 & -0.00215124 \\ 0.09377284 & -0.00269751 \\ -0.59357227 & -0.00520447 \\ 0.90372127 & -0.00361066 \\ 0.88684858 & -0.00013831 \\ -0.45472543 & -0.00071932 \\ -0.94887294 & 0.00006135 \\ -0.00691795 & 0.00076317 \\ 1.81478200 & 0.00051859 \\ 1.39689516 & 0.00062758 \\ 1.16065673 & -0.00253060 \\ 1.20861349 & -0.00067408 \\ -1.47964282 & -0.00419727 \\ -1.38265045 & -0.00261656 \\ -0.83112063 & 0.87235110\end{array}$


$\mathrm{H}$
$\mathrm{H}$
$\mathrm{H}$
$\mathrm{H}$
$\mathrm{O}$

$\mathrm{S}_{0}$ state of keto-s-cis(-1)

C

C

C

C

C

C

C

C

C

C

$\mathrm{N}$

$\mathrm{N}$

$\mathrm{S}$

$\mathrm{S}$

$\mathrm{H}$

$\mathrm{H}$

$\mathrm{H}$

$\mathrm{H}$

$\mathrm{H}$

$\mathrm{O}$

$\mathrm{O}$

$\mathrm{S}_{1}$ state of keto-s-twist(-1)
4.89072771

$-3.52329735$

$-2.48614928$

$-4.82764779$

4.82475472

$-5.57628973$

0.44556921

1.82099520

4.29056730

3.88859620

$-1.61773994$

$-1.99194393$

$-3.28314508$

$-4.35843038$

$-2.67778605$

$-3.95650557$

2.55388869

$-0.35390120$

2.74724156

$-0.51255530$

4.88385204

4.88136361

$-3.57411388$

$-2.39155256$

$-4.76056578$

4.75843570

$-5.53648232$

$-0.42131900$

$-1.87229283$

$-4.16705351$

$-4.06310940$

1.62215172

1.94269548

3.27702602

4.37846360

2.70655188

3.99150765

$-2.75591190$

0.28389700
$-0.82968354$

$-1.99921212$

2.86415515

2.10709964

1.73302027

$-0.39563703$

0.02311822

0.08155437

$-0.54877136$

0.94116195

0.84177633

$-0.57189153$

$-0.97652810$

0.02518282

1.82990971

1.45046744

1.16727083

1.12228305

$-1.47236938$

$-1.50550148$

$-0.76792129$

$-0.76534950$

$-2.01077474$

2.86643510

2.16454765

1.78150363

$-0.29345745$

$$
\begin{array}{r}
-0.00148948 \\
-0.00270112 \\
-0.00625466 \\
-0.00319470 \\
0.00064195 \\
-0.00029136 \\
-0.00037255 \\
0.00003697 \\
0.00121878 \\
0.00089805 \\
-0.00151153 \\
-0.00002795 \\
-0.00556378 \\
-0.00152163 \\
0.87112015 \\
-0.88594993 \\
-0.00118386 \\
0.00167799 \\
0.00133061 \\
-0.00136711 \\
-0.00050257
\end{array}
$$

$-0.13002777$

$-0.47882721$

$-0.10303225$

$-0.25418405$

0.14668113

0.82187023

$-0.31807658$

$-0.63485797$

$-0.83402751$

0.00087614

0.48440077

$-0.28353294$

0.97399353

$-0.25739238$

0.07902531

0.09512266

$-1.72008294$

0.33471459

$-1.29308981$

0.38262754

$-0.40100740$

$-1.11336261$

$-1.19253336$

$-0.01065081$ 
$\mathrm{S}_{0}$ state of keto-s-twist(-1)

$\mathrm{C}$
$\mathrm{C}$
$\mathrm{C}$
$\mathrm{C}$
$\mathrm{C}$
$\mathrm{C}$
$\mathrm{C}$
$\mathrm{C}$
$\mathrm{C}$
$\mathrm{C}$
$\mathrm{N}$
$\mathrm{N}$
$\mathrm{S}$
$\mathrm{S}$
$\mathrm{H}$
$\mathrm{H}$
$\mathrm{H}$
$\mathrm{H}$
$\mathrm{H}$
$\mathrm{O}$
$\mathrm{O}$

$\mathrm{S}_{1}$ stat of keto-s-trans(-1)

C
C
C
C
C
C
C
C
C

$-2.47513749$

0.37888650

0.45400964

1.40492597

1.38134921

$-4.68467824$

$-0.60862685$

1.06360685

1.98940747

$-2.74454605$

$-1.96965353$

$-0.57263371$

0.47313631

5.54007201

$-0.40725687$

$-1.88831657$

$-4.27774318$

$-3.98581914$

1.65077401

2.00222205

3.30095432

4.39552580

2.69016666

3.99475262

$-2.62062155$

0.29298389

$-2.67145110$

0.47205202

$-4.82866418$

$-4.87829139$

3.54234407

2.44616485

4.79249409

$-4.85153888$

5.58200736

$-0.42429609$

$-1.87028300$

$-4.28694073$

$-3.93147044$

1.68834980

1.92590940

3.24100251

4.36336934

2.78403239
$-0.04241603$

$-0.06649990$

0.13453374

$-0.36408597$

$-0.80706167$

0.51913763

0.95910928

0.03971863

$-1.71774201$

$-1.30988619$

$-0.42339157$

$-1.08160107$

0.44653234

1.45289294

$-0.62388411$

1.03205680

1.97891700

$-2.73859936$

$-2.01153008$

$-0.66098741$

0.39151311
$-0.62954358$

1.39758672

0.86031378

$-0.51058767$

0.55371720

0.63568869

$-1.27982678$

0.12401594
-0.08618636
-0.03492543
0.72335780
-0.69750913
0.11958771
-0.19625225
-0.29479690
-0.06289212
0.34782258
0.25967029
-1.00036722
0.17978767
1.47470146
-0.43469028
1.26177059
0.67159405
-0.53615404
0.59108861
0.43498261
-1.45060778
-0.13486518 


\begin{tabular}{|c|c|c|c|}
\hline $\mathrm{C}$ & 4.05687314 & 1.22002074 & -0.00015925 \\
\hline $\mathrm{N}$ & -2.59672402 & -0.96660656 & -0.00090393 \\
\hline $\mathrm{N}$ & 0.31787864 & 1.16704693 & 0.00057582 \\
\hline S & -2.71950785 & 1.67372478 & -0.00050464 \\
\hline $\mathrm{S}$ & 0.41957442 & -1.44905128 & -0.00056794 \\
\hline $\mathrm{H}$ & -4.87780031 & 1.00442874 & 0.87556617 \\
\hline $\mathrm{H}$ & -4.87669684 & 1.00477456 & -0.87937191 \\
\hline $\mathrm{H}$ & 3.42529384 & -2.15979319 & -0.00135728 \\
\hline $\mathrm{H}$ & 2.58374320 & 2.74440524 & 0.00078945 \\
\hline $\mathrm{H}$ & 4.89471115 & 1.89416980 & -0.00018227 \\
\hline $\mathrm{O}$ & -4.80796915 & -1.54996531 & -0.00231349 \\
\hline $\mathrm{O}$ & 5.53732760 & -0.62666006 & -0.00120240 \\
\hline \multicolumn{4}{|c|}{$\mathrm{S}_{0}$ state of keto-s-trans $(-1)$} \\
\hline $\mathrm{C}$ & -0.44685085 & -0.00472561 & 0.00095913 \\
\hline $\mathrm{C}$ & -1.81367653 & 0.06443774 & -0.00034168 \\
\hline $\mathrm{C}$ & -4.22523061 & 0.82487836 & -0.00153908 \\
\hline $\mathrm{C}$ & -3.92324321 & -0.69159966 & -0.00189349 \\
\hline $\mathrm{C}$ & 1.63479171 & 0.74726864 & 0.00042011 \\
\hline $\mathrm{C}$ & 1.95085337 & -0.67944171 & 0.00004104 \\
\hline $\mathrm{C}$ & 3.22926530 & -1.12586896 & -0.00063326 \\
\hline $\mathrm{C}$ & 4.33844058 & -0.16335893 & -0.00071449 \\
\hline $\mathrm{C}$ & 2.72735715 & 1.70184308 & 0.00028191 \\
\hline $\mathrm{C}$ & 3.99019821 & 1.27705087 & -0.00039808 \\
\hline $\mathrm{N}$ & -2.61829448 & -0.99307305 & -0.00143100 \\
\hline $\mathrm{N}$ & 0.37794727 & 1.07250441 & 0.00047352 \\
\hline S & -2.63138075 & 1.66697680 & -0.00074974 \\
\hline S & 0.43664838 & -1.56360231 & 0.00020648 \\
\hline $\mathrm{H}$ & -4.80812060 & 1.07089190 & 0.87603275 \\
\hline $\mathrm{H}$ & -4.80741877 & 1.07139460 & -0.87943799 \\
\hline $\mathrm{H}$ & 3.48429117 & -2.16946521 & -0.00155100 \\
\hline $\mathrm{H}$ & 2.47745279 & 2.74770375 & 0.00066180 \\
\hline $\mathrm{H}$ & 4.82008915 & 1.96075516 & -0.00089676 \\
\hline $\mathrm{O}$ & -4.85202962 & -1.46369547 & -0.00257667 \\
\hline $\mathrm{O}$ & 5.50528734 & -0.52096221 & -0.00110319 \\
\hline
\end{tabular}

